\title{
Multi-objective optimization of a flash lamp drive
}

\author{
R. Gelagaev, J. Zwysen, K. Engelen, P. Tant, F. Bamelis, T. Geurts and J. Driesen \\ Katholieke Universiteit Leuven, Esat-Electa \\ Kasteelpark Arenberg 10/2445 \\ Heverlee, Belgium \\ E-Mail: ratmir.gelagaev@esat.kuleuven.be \\ URL: http://www.esat.kuleuven.be/electa
}

\section{Keywords}

«Power management», «Regulation», «Lamp», «Optimization», «Genetic algorithm».

\begin{abstract}
To adjust the light spectrum of a flash lamp, the current flowing through the flash lamp has to be controlled. An optimal design of a switched resistor bank circuit following a predefined current profile is presented. The optimal values of the switched resistors are calculated using multi-objective optimization with genetic algorithms.
\end{abstract}

\section{Introduction}

Flash lamps are widely used in many industrial, medical and analytical applications ex. solar simulators and water inspection systems. They can produce instant high-power light pulses or provide a high-intensity continuous spectrum. A flash lamp is typically ignited by a capacitor discharge. Fig. 1 shows a typical power supply for flash lamps, [1]. It consists of a charging circuit (voltage multiplier), bleed resistors, a capacitor bank and a trigger circuit.

The charging circuit charges the capacitors to a specific voltage. A large current flows through a discharged capacitor as it is equivalent to a short circuit. Therefore, a current-limiting device is included in the charging circuit for its protection. The current can be limited by a series inductance or a resonant charging circuit [2].

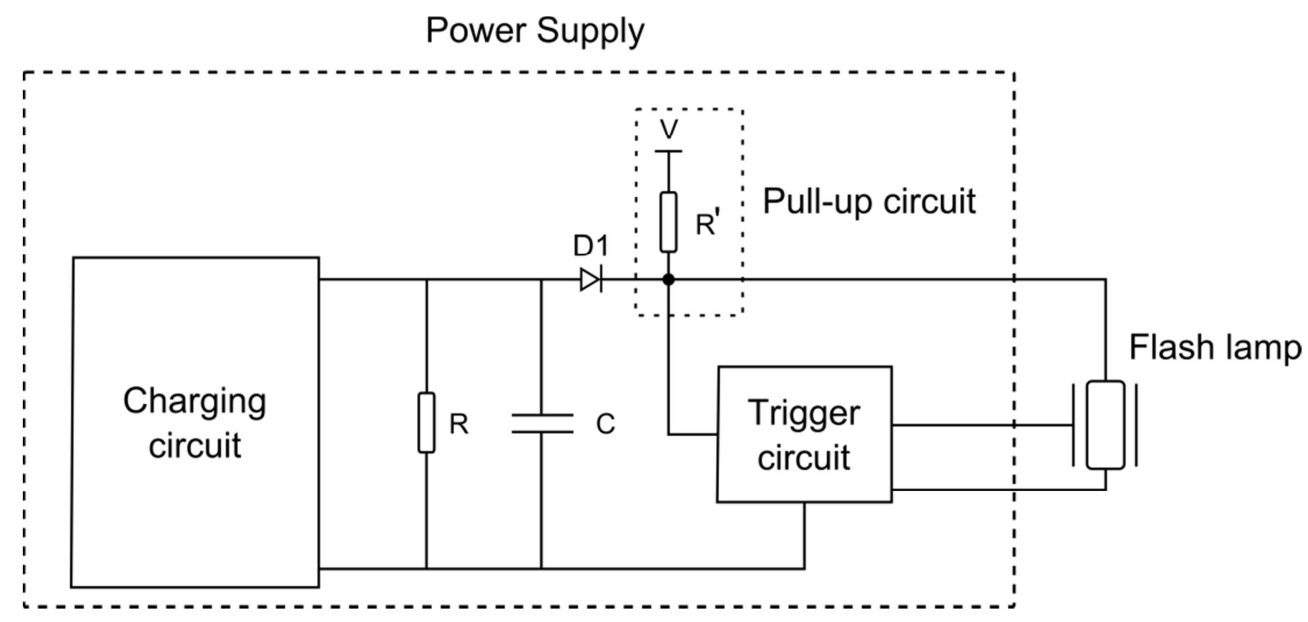

Fig. 1: Power supply for flash lamps

A pull-up circuit, represented by a resistor, ensures that a high voltage is applied across the flash lamp before the trigger pulse is applied. The diode prevents the current from the pull-up circuit from 
flowing into the capacitors. The trigger circuit initiates the discharge of the stored energy into the flash lamp by applying a high-voltage pulse.

During the discharge of the capacitors, the flash lamp produces a light pulse that depends on the stored capacitor energy. In order to shape the light intensity profile and the spectrum of the light pulse produced by the flash lamp, the discharge of the stored capacitor energy, i.e. the current flowing through the lamp, has to be controlled in some way.

The first part of the paper describes the measurement and modeling procedure of the flash lamp. Then two circuits for current shaping in flash lamps are described together with their advantages and disadvantages. After that the presented resistor bank circuit is explained. In the next section the presented circuit is optimized using genetic algorithm NSGA-II. Finally, the proof of concept is shown with some measurements followed by the conclusion.

\section{Modeling of the flash lamp}

In order to optimize the circuitry the flash lamp has to be modeled with measurements. The measurement setup (Fig. 2) consists of a power supply, a flash lamp, a light sensor, a scope and measurement probes. The used scope is a Tektronix TDS 5054 500Mhz. The current through the flash lamp is measured with a PEM Rogowski Current Waveform Transducer. The voltage across the flash lamp is measured with a TESTEC TT-SI 9010A 70MHz High Voltage Differential Probe.

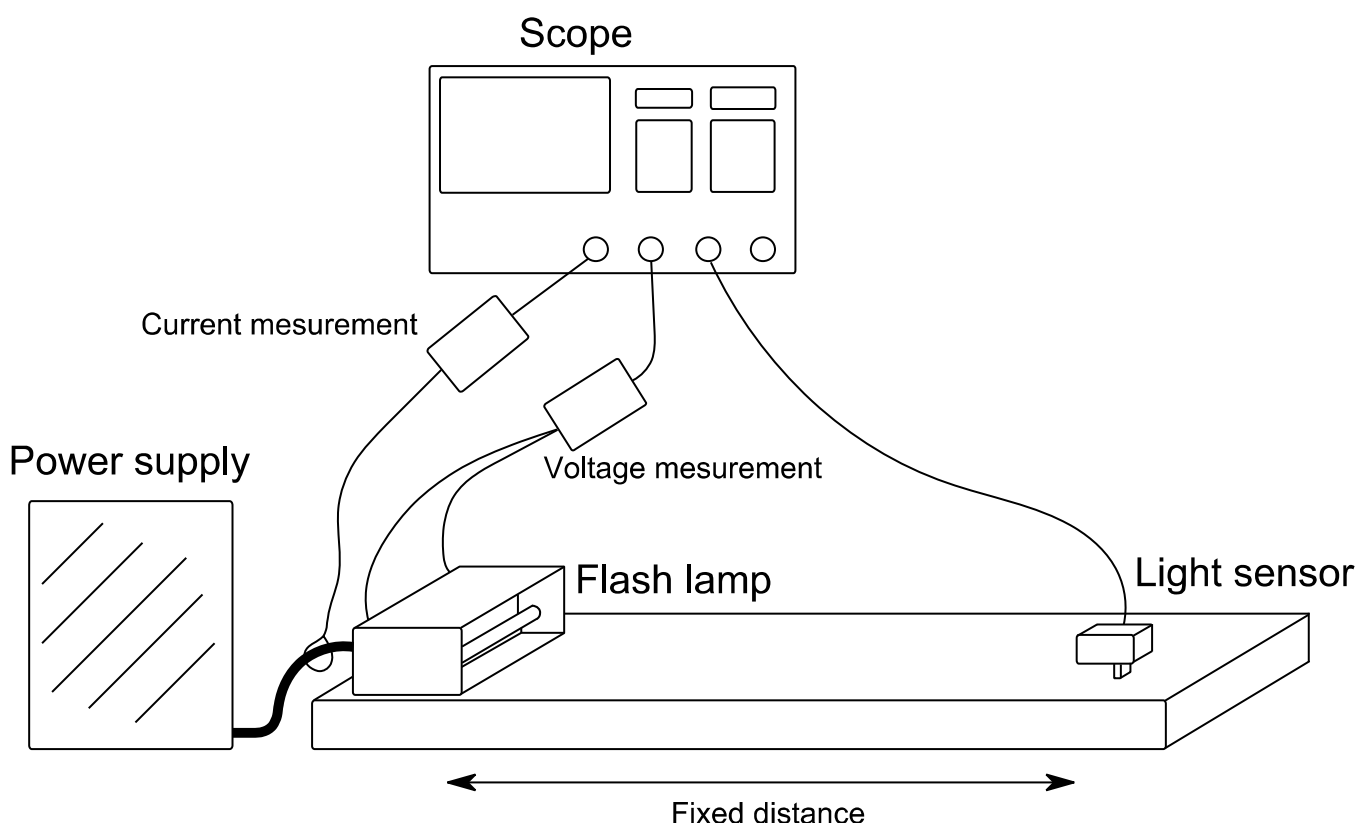

Fig. 2: Measurement setup

The distance between the lamp and the light sensor should be kept constant to ensure a specific relationship between the voltage of the capacitor bank and the corresponding light profile. The output voltage of the light sensor is proportional to the measured light intensity.

The circuitry of the power supply is shown in Fig. 1. When the flash lamp is ignited by the trigger circuit, a large current flows through the flash lamp. The peak value of the current can exceed 1000A. However, this peak current lasts only for a few milliseconds as the capacitor bank is discharged instantaneously.

To make the simulations and the optimization easier, the flash lamp is modeled as a resistor. This is justified by the fact that during the first $15 \mathrm{~ms}$ the flash lamp has an ohmic behavior. The value of the resistance was measured to be $0.6 \mathrm{Ohm}$. Furthermore, it is very difficult to set up a realistic simulation of a setup with the light measured by a sensor. Therefore, the light profile of the flash lamp is replaced 
by its corresponding current profile. The measurements (Fig. 3) show that there is a quasi-linear relationship between the light produced by the flash lamp and the current flowing through it.

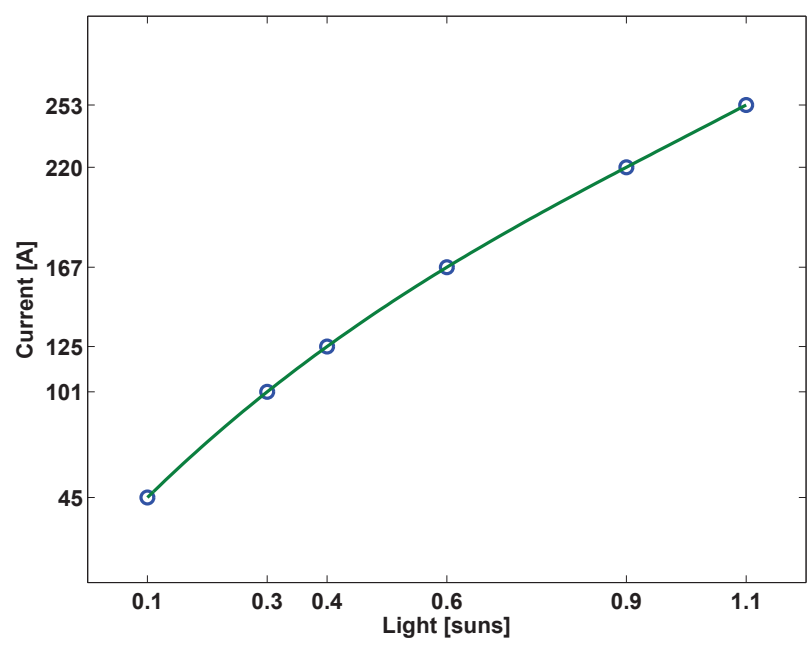

Fig. 3: Quasi-linear relationship between the light intensity and the current through the flash lamp

\section{Circuits for current shaping in flash lamps}

\section{Series inductance}

Since there is a quasi-linear relationship between the current and the light intensity, it is possible to use an inductor to extend the current flow in time in order to extend the light pulse duration, Fig.4. The trigger circuit, pull-up circuit and the diode are omitted for the sake of clarity. Diode D2 is an external freewheeling diode. Ideally, the series inductance does not contribute to any losses. Therefore, it can be assumed that all energy from the capacitor bank will be transferred to the flash lamp.

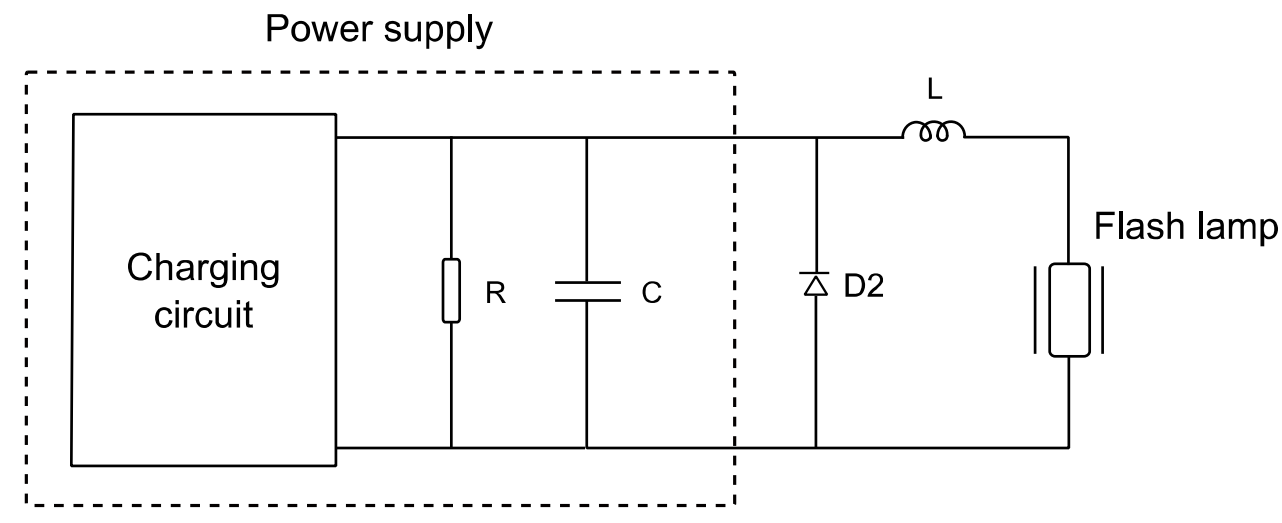

Fig. 4: Series inductance

However, the main problem here is the saturation of the inductor due to the high currents involved. It is also not possible to shape any complicated current profile other than an exponentially decreasing current profile. Another disadvantage of using coils is that they are big, heavy, noisy and expensive.

\section{Switched mode power supply}

The best solution for the problem is a switched mode power supply. The current through the flash lamp is measured and controlled by adjusting the duty cycle. Because there are almost no losses in this circuit, like with the series inductance, all of the energy is used in the flash lamp making its light pulse last longer. Furthermore, it is also possible to shape complicated current profile using a switched mode power supply. However, this option is only useful for high demanding applications because of the extremely high peak power level $(>100 \mathrm{KW})$. 


\section{Switched resistor bank (presented circuit)}

The presented circuit consists of a switched resistor bank that controls the current through the flash lamp, Fig. 5.

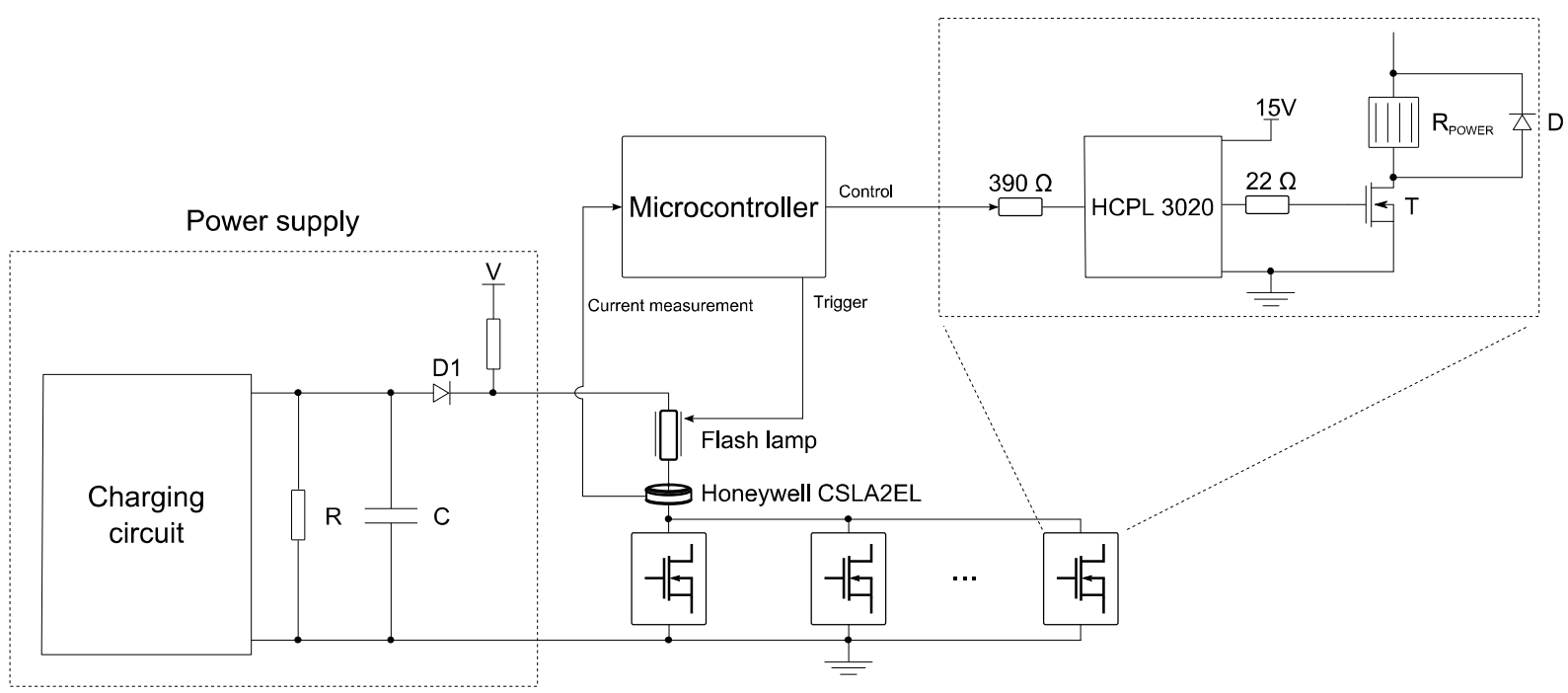

Fig. 5: Switched resistor bank

The flash lamp is triggered by the microcontroller and the current is measured with the Honeywell CSLA2EL current sensor, the output of which is connected to the microcontroller. If the measured current is different from the desired current profile, the parallel resistors are switched on/off to adjust the current value. Each IGBT is controlled by an isolated gate driver (HCPL3020) and is switched on by a $5 \mathrm{~V}$ control signal from the microcontroller. This is repeated $\mathrm{N}$ times in parallel.

The design of the optimized circuit is depicted in Fig. 6 . There are 24 power resistor mounted on 6 metal plates that also serve as a heat sink. Each resistor is controlled separately by the microcontroller (PIC18F4550). The current sensor provides a real-time current measurement through the flash lamp. The resistors are switched on and off to follow a predefined current profile.

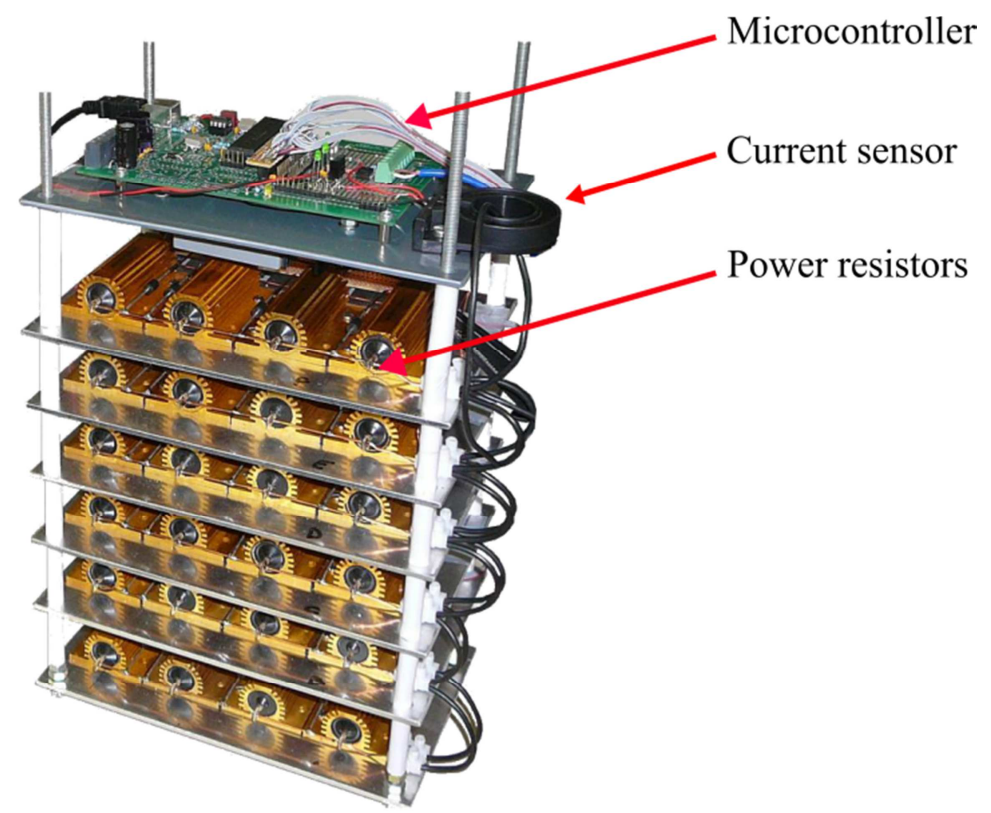

Fig. 6: Design of the switched resistor bank circuit 


\section{Multi-objective optimization of the switched resistor bank}

For the optimization, a system with $N$ identical power resistors with a resistance of $R$, each of which can be connected in parallel, is assumed. The lamp is modeled by a resistor with a constant resistance, $R_{\text {lamp. }}$. The digital controller, with sampling time $T_{s}$, sets the number of resistors connected in parallel in each time interval $n_{i}$. This number is chosen in such a way that the maximum deviation of the current through the lamp, $i_{\text {lamp }}$, from the reference current, $i_{\text {ref }}$, is minimized. At the start of the i-th time interval, the voltage, $V_{i}$, across the capacitor bank, with capacitance $C$, is sampled. From this, the current at the start of the interval, $I_{i}$, and the current drop during the interval, $\Delta I_{i}$, can be calculated using (1) and (2). The controller then selects $n_{i}$ to minimize (3). All the aforementioned definitions are shown in Fig. 7.

$$
\begin{aligned}
& I_{i}=\frac{V_{i}}{R} n_{i} \\
& \Delta I_{i}=\frac{V_{i}}{R} n_{i}\left(1-e^{-\frac{T_{s}}{R C} n_{i}}\right) \\
& \left|\left(I_{i}-\frac{\Delta I_{i}}{2}\right)-i_{r e f}\right|_{t=\frac{t_{i}+t_{i+1}}{2}} \mid
\end{aligned}
$$
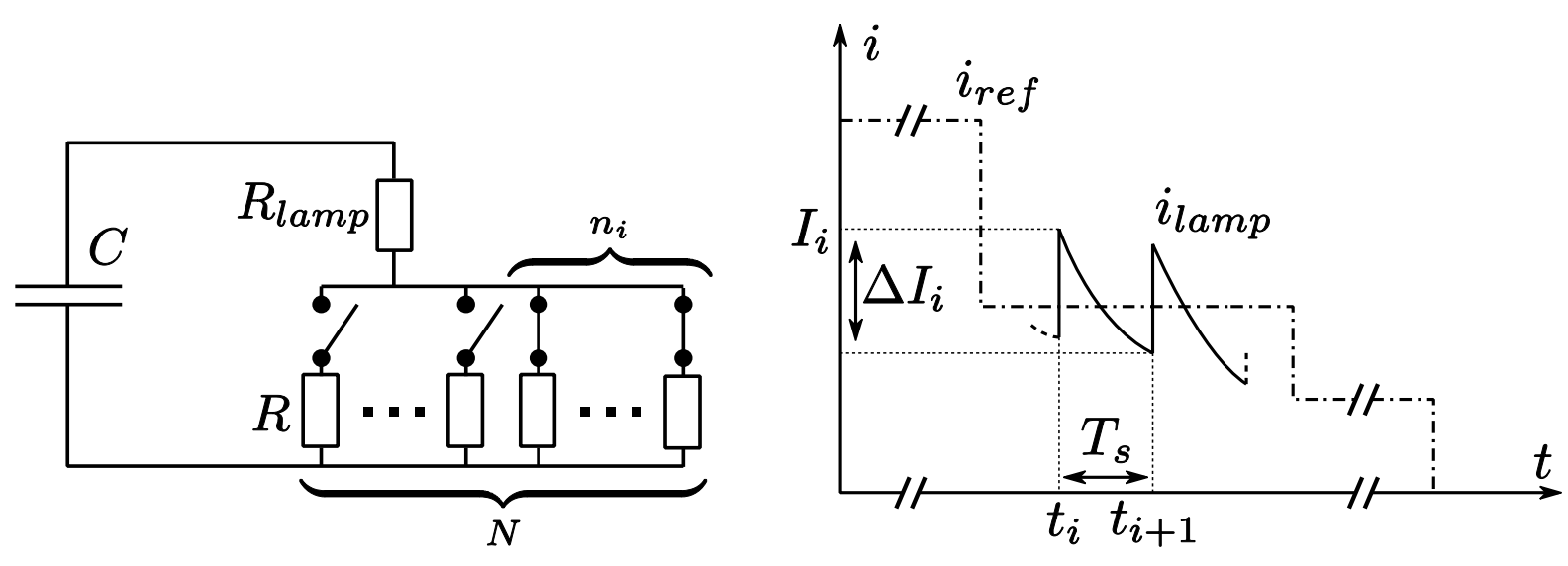

Fig. 7: Visualization definitions

A multi-objective algorithm is used to determine the optimal number of parallel resistors, $N$, the resistance of the power resistors, $R$, and the starting voltage on the capacitor bank, $V_{0}$. There are two main objectives: the total energy loss in the power resistors, $E_{R}$, and the maximum deviation of the current through the lamp from the reference current, $\Delta I_{\max }(4)$.

$$
\operatorname{minimize}\left\{\begin{array}{c}
E_{R}=\sum_{i} \int_{t_{i}}^{t_{i+1}} \frac{R}{n_{i}} i_{\text {lamp }}^{2}(t) d t \\
\Delta I_{\max }=\max \left|i_{\text {lamp }}(t)-i_{\text {ref }}(t)\right|
\end{array}\right.
$$

The power resistors will be chosen from the E12 range, which means that both $N$ and $R$ are discrete parameters. A genetic algorithm is well suited for multi-objective optimization with a mix of discrete and continuous parameters [3]. The genetic algorithm NSGA-II was selected and implemented [4]. Intermediate crossover and Gaussian mutation was used to create each next generation. The limits on each parameter, as well as the parameters for crossover and mutation, are given in table I. Table II gives the values of the other parameters that are constant during the optimization. 
Table I: Optimization Parameters

\begin{tabular}{|l|l|l|l|}
\hline & Limits & a & o \\
\hline $\boldsymbol{N}$ & $1 . .50$ & 0.2 & 3 \\
\hline $\boldsymbol{R}$ & $0.1 . .820 \Omega$ & 0.2 & 50 \\
\hline $\boldsymbol{V}_{\boldsymbol{0}}$ & $300 . .1000 \mathrm{~V}$ & 0.2 & 3 \\
\hline
\end{tabular}

Table II: Other Parameters

\begin{tabular}{|l|l|}
\hline Parameter & Value \\
\hline $\boldsymbol{C}$ & $9.3 \mathrm{mF}$ \\
\hline $\boldsymbol{T}_{\boldsymbol{s}}$ & $50 \mu \mathrm{s}$ \\
\hline $\boldsymbol{R}_{\text {lamp }}$ & $0.6 \Omega$ \\
\hline $\boldsymbol{I}_{\text {ref }}(\boldsymbol{t})$ & $\left\{\begin{array}{l}400 \mathrm{~A}, 0 \mathrm{~ms} \leq t<6 \mathrm{~ms} \\
200 \mathrm{~A}, 6 \mathrm{~ms} \leq t<12 \mathrm{~ms} \\
100 \mathrm{~A}, 12 \mathrm{~ms} \leq t<18 \mathrm{~ms}\end{array}\right.$ \\
\hline
\end{tabular}

In the first optimization, the three parameters $N, R$ and $V_{0}$ are optimized towards the two main objectives, $E_{R}$ and $\Delta I_{\max }$, and towards a third objective, $N$. So the number of resistors in parallel is also minimized. The result of that optimization is shown as color maps in Fig. 8, 9 and 10. Fig. 8 shows the parameter and objective, $N$. Fig. 9 and Fig. 10 show the parameters $R$ and $V_{0}$ respectively. For the color maps, an interpolation between the 10000 solutions is used for clearer visualization. The black lines in Fig. 8, 9 and 10 are the result of three additional optimizations where parameter $N$ is held constant at 10,20 and 30. For those optimizations, only $R$ and $V_{0}$ were optimized towards $E_{R}$ and $\Delta I_{\max }$.

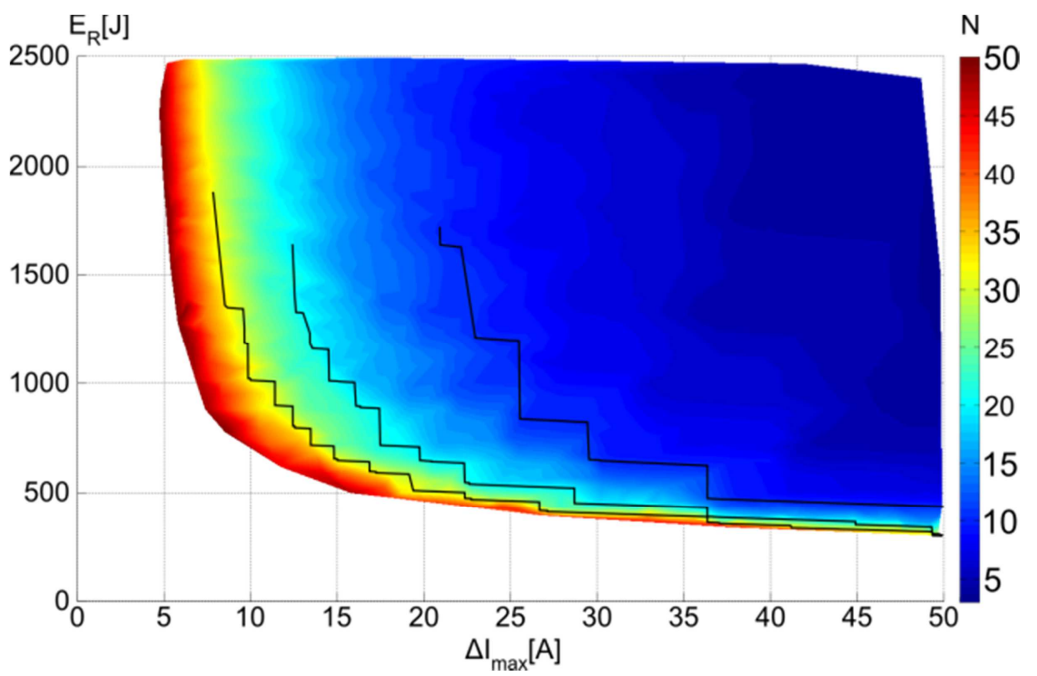

Fig. 8: Results optimization, the three objectives, $E_{R}, \Delta I_{\max }$ and $N$, are shown

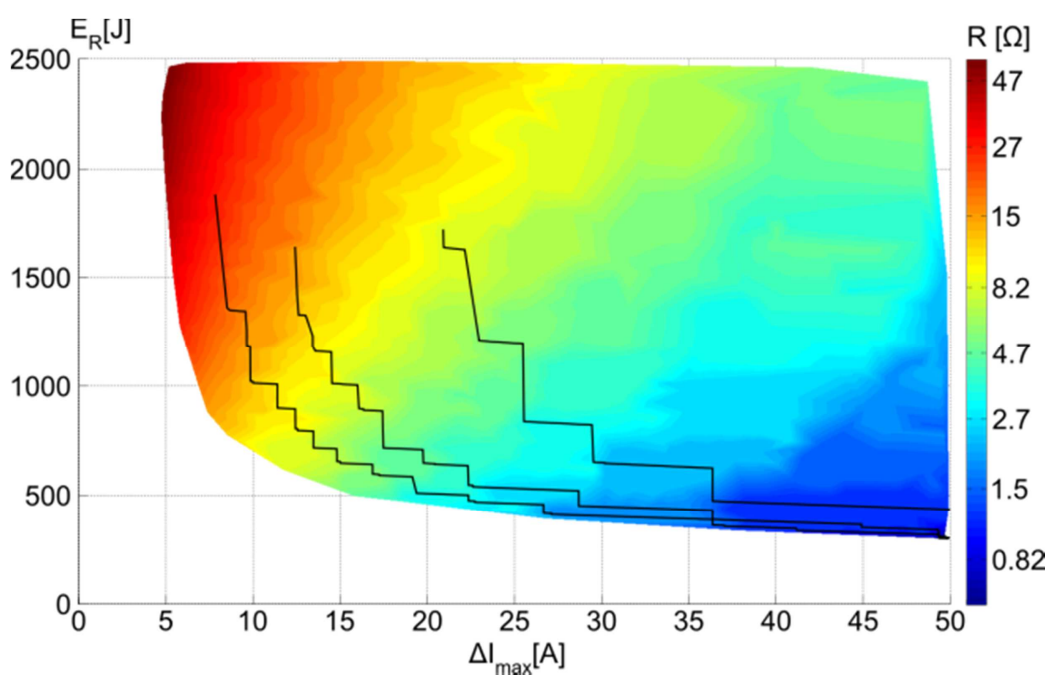

Fig. 9: Results optimization, the two main objectives, $E_{R}$ and $\Delta I_{\max }$, and parameter $R$ are shown 


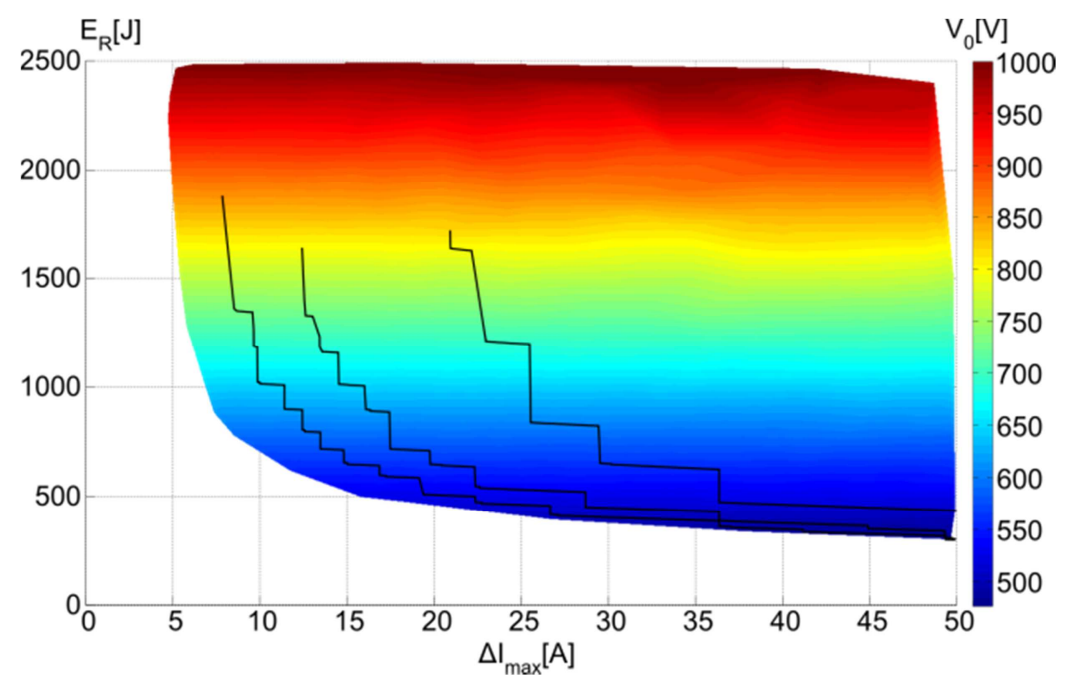

Fig. 10: Results optimization, the two main objectives, $E_{R}$ and $\Delta I_{\max }$, and parameter $V_{0}$ are shown

From Fig. 8, it is clear that a greater number of parallel resistors allow for smaller energy losses and maximum deviations of the current. Fig. 9 shows that smaller resistances are chosen to minimize energy loss in the power resistors while larger resistances are used to minimize the maximum current deviation. From Fig. 10, it is clear that a higher initial voltage on the capacitor bank leads to higher losses in the resistors. From this optimization, 20 power resistors with a resistance of $4.7 \Omega$ is a good trade-off between the number of parallel resistances and the maximum deviation of the current through the lamp.

\section{Measurement results}

The switched resistor bank circuit is been tested to follow three different current levels: 400A, 200A and $100 \mathrm{~A}$ each lasting for $6 \mathrm{~ms}$, Fig. 11. Before the trigger the voltage across the capacitor is kept high by the pull-up circuit. Immediately after the trigger pulse, the switched resistor bank starts to switch on resistors in decreasing order. Once the desired current value has been established, the circuit waits until the current decreases further before switching on any additional resistors. The voltage across the capacitors drops linearly. The steepness of the drop depends on the value of the current level.
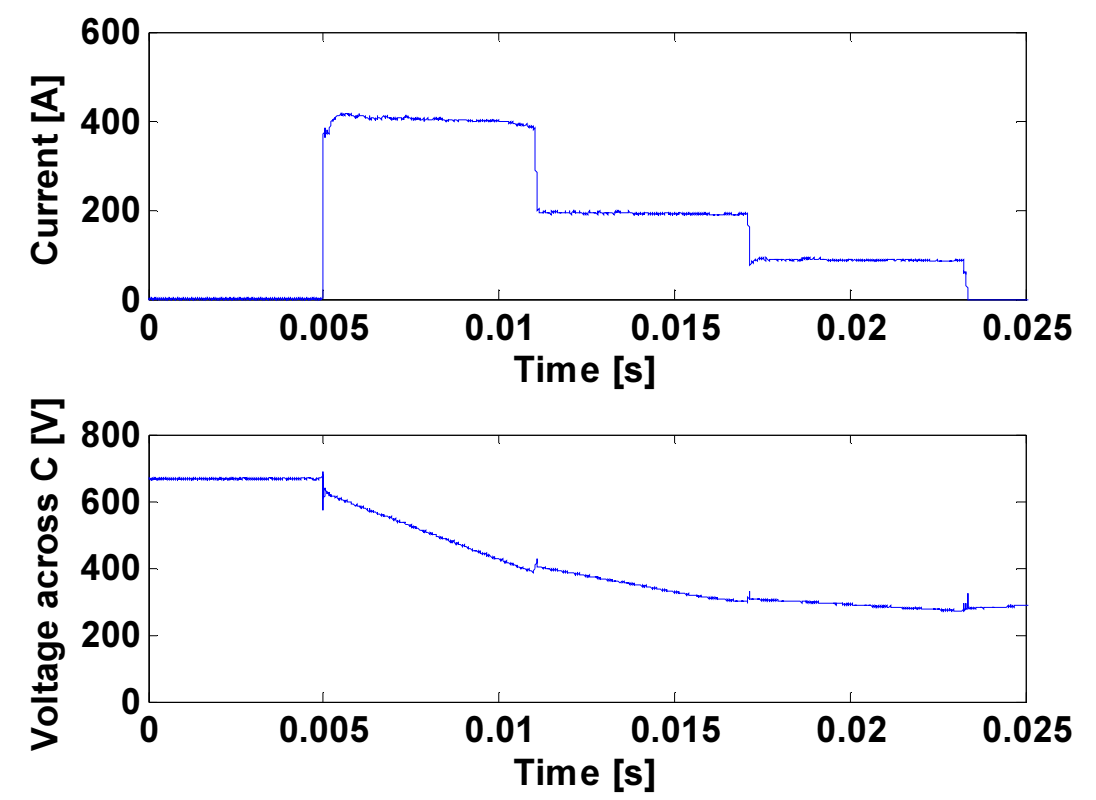

Fig. 11: Measurement results 


\section{Conclusion}

This paper presents an optimal design of a switched resistor bank circuit following a predefined current profile. A flash lamp is used as an application for the presented circuit.

The flash lamp is measured and modeled as a resistor. The measurements also show that there is a quasi-linear relationship between the light intensity and the current through the flash lamp.

Next to the presented switched resistor bank circuit, two alternative circuits have been discussed with their advantages and disadvantages. This also explains why the presented switched resistor bank circuit is the right choice.

A genetic algorithm based on NSGA-II was used for the multi-objective optimization of the presented circuit. Using the Pareto plots, the optimal number of the resistors and their values were determined. These optimal values were used to build the presented switched resistor bank circuit and the measurements show a close similarity with the values calculated in the optimization procedure.

\section{References}

[1] W. Koechner, Solid-state laser engineering, 6th ed. Springer, 2006, pp. 315-323

[2] D.W. McDonald, "Design, control and fault protection of resonant charging circuits for high frequency laser pulse modulators," in proc. IEE Colloquium on Pulsed Power Technology, 20 Feb 1992.

[3] A. Konak, D. W. Coit and A. E. Smith, "Multi-Objective Optimization Using Genetic Algorithms: A Tutorial”, Elsevier Journal on Reliability Engineering \& System Safety, vol. 91, nr. 9, Sep. 2006, pp. $992-1007$.

[4] K. Deb, A. Pratap, S. Agarwal, and T. Meyarivan, "A fast and elitist multiobjective genetic algorithm: NSGA-II,” IEEE Trans. Evol. Comput., vol. 6, no. 2, April 2002, pp. 182-197 